\title{
Innovation ability and innovation spirit in photoelectric comprehensive experiment teaching
}

Dexing Wang, Yang Zhang, Xiaofeng Wang, Xiaojun Zhang, Tao Zhang, et al.

Dexing Wang, Yang Zhang, Xiaofeng Wang, Xiaojun Zhang, Tao Zhang, Peng Sun, "Innovation ability and innovation spirit in photoelectric comprehensive experiment teaching," Proc. SPIE 10452, 14th Conference on Education and Training in Optics and Photonics: ETOP 2017, 104526F (16 August 2017); doi: $10.1117 / 12.2269942$

SPIE Event: 14th Conference on Education and Training in Optics and Photonics, ETOP 2017, 2017, Hangzhou, China 


\title{
Innovation ability and innovation spirit in photoelectric comprehensive experiment teaching
}

\author{
Wang Dexing, Zhang Yang ${ }^{*}$, Wang Xiaofeng, Zhang xiaojun, Zhang Tao, Sun Peng \\ Key Laboratory of integrated fiber optics, Ministry of education. \\ National Demonstration Center for Physical Experiment Education, Harbin \\ Engineering University, China.
}

\begin{abstract}
The traditional experimental teaching methods have some shortcomings in the training the student innovation ability. In order to improve the student practical ability in the photoelectric technology, in this paper new experimental teaching modes are tried and reformed for cultivating the innovative ability of students in the linear CCD experiment. The photoelectric experiment systems are independently designed and completed by students. Compared with the traditional experimental teaching methods, this new methods have a great role in the development of the ability of creative thinking.
\end{abstract}

Keywords: Innovation ability, innovation spirit, teaching methods, photoelectric technology

Photoelectric comprehensive experiment is the core of a professional experimental course in photoelectric information and science engineering major ${ }^{1,2}$. And it is an important practical teaching part of theoretical courses in photoelectric technique. The contents of experiment include photoelectric sensing technology, photoelectric detection technology, optical information processing and infrared technology and other basic experiments. This experimental curriculum has very important effect about how to improve student's practical operating abilities, thought ability to innovate and ability to understand theoretical knowledge. In line with the rapid development of optoelectronic information technology on the needs of personnel training ${ }^{3-5}$.

\section{The idea that how to cultivate innovative ability in experimental teaching.}

The main contents of photoelectric comprehensive experiment are photoelectric sensor principle, measurement of device characteristic parameter and typical application experiment.

The traditional experimental teaching methods usually adopt existing experimental apparatus to wire and operate simply on the experimental apparatus which have been designed and debugged. Measure the result of the experiment and complete the experiment report to verify the theory of knowledge has been studied.

Apparently, students have little gain through the completion of such experiment. Teaching practice should be to cultivate students hands-on, brain ability and comprehensive use of knowledge and ability to solve practical problems as the main purpose. To this end, we should create an open experimental condition and environment that allows students or instructors to design their own experimental content, build their own experimental system, perform more experimental choices. According to the purpose of the experiment and the requirements of their own structures, students should assemble experimental systems, complete the experimental content. It's better to improve the ability of students than through the black box which has been designed. In this way, students can achieve a multiplier effect. Students can promote in-depth understanding and awareness on the use of the instrument principle, structure, as well as the system parameters of the impact of the experimental results and other aspects through build experimental system by themselves. Meanwhile, the construction of the experimental system can cause students' interest in learning, experimental results and possible experimental data will give students the opportunity to use their brain and think in order to in-depth study about how to improve the

14th Conference on Education and Training in Optics and Photonics: ETOP 2017, edited by Xu Liu,

Xi-Cheng Zhang, Proc. of SPIE Vol. 10452, 104526F · @ 2017 ICO, IEEE, OSA, SPIE

CCC code: $0277-786 \mathrm{X} / 17 / \$ 18 \cdot$ doi: $10.1117 / 12.2269942$ 
measurement accuracy, measurement range and stability the experimental method. After the success of the experiment, students can appreciate comfort and happiness through the results. In addition, in the open experimental platform not only need to build their own experimental system, it can also design their own experimental questions and content to fully develop students and instructors innovative ideas, mobilize the enthusiasm of students to take the initiative to learn and cultivate and improve the ability to think. The most important thing is to improve the practical ability of students to adapt to the needs of social development and technological progress for setting up a solid foundation for entrepreneurship and career development to open up a broader space in the future.

\section{Experimental teaching of cultivating innovation ability}

\subsection{Emphasize the experimental operation}

The traditional experimental teaching is based on the use of integrated experimental box to repeat the operation and verification on the theoretical teaching of the classic experiments. The experimental box is designed for an experimental purpose without changing the instrument. It don't need students to design experimental programs and build experimental devices. In the experiment students only need to complete a simple connection, then they can use the experimental instrument to adjust the experimental phenomenon, record and deal with experimental data. It is difficult to really exercise innovation and experimental design capabilities. As the saying goes, see it again as it is. Let the students use their brain, hands to build their own experimental system, and then complete the experiment by measurement system according to the required steps. As a result, students benefit not only to complete the experimental task itself, more importantly, to build the experimental process of using brain and improving hands-on ability to exercise. For example, the non - contact experiment which uses linear array CCD to measure object dimension. Students should connect the telecentric light source circuit to complete optical system optical system the light source, the measured object, imaging objective lens and CCD camera layout and debugging. Through adjust the object distance, focal length and aperture and other parameters to observe the waveform easily. Through their own measurement system, students can easily analyze and find the factors that improve the measurement accuracy, expand the measurement range and enhance the stability and other aspects of the factors and methods. Especially in the process of building the system, if students encountered difficulties and failures, their experimental skills will get better in the solution of difficulties and failures in the process. After the specific experimental process of training, students will improve abilities to use their brain and hand obviously. So that they can learn to understand the laws of science, master the idea of instrument design and learn modern technology, for their future work to lay a firm foundation.

\subsection{Strengthen the comprehensive application of experimental content into the experimental teaching courses}

The types of experiments often include validation experiments, comprehensive experiments and design experiments and other experiments. Validation experiments content is single and dull, often to verify the theory just learned for the purpose, can not immediately see its practical significance. It is difficult to cause students a strong interest, the experimental results is not so good. But the validation experiments is the basic practice of students to master the device, the basic process of the experiment, and the indispensable part in the whole process. For example, the experiment of the characteristic of conventional LED/LD, photovoltaic detectors principle of linear array CCD and experiment of driving circuit in photoelectric technology experiment. These experiments can be arranged in the early stages of experimental teaching, So that students are fully familiar with the various parts of the photoelectric experimental platform and usage of the light source, detection device performance in the light detection 
technology. The comprehensive experiment can be set up after students can understand the performance of the basic device and bias circuit. Comprehensive experiment aims to use multiple courses of knowledge, and a wide range of knowledge and methods to solve problems, to develop students to use the knowledge, experimental methods and experimental skills and improve the ability to analyze and solve problems. The use of comprehensive experiments can be integrated with the relevant theoretical teaching content, and the introduction of practical, closely related with life and new technologies, new applications for the experimental content, so that the experimental content in line with the scientific development and practical needs of the photovoltaic industry. Not only students can learn to use and cause students a strong interest, more important is to broaden the students to learn professional knowledge, improve students' ability to solve practical problems, enhance students to adapt to social development and scientific and technological progress requirements. For example, after completing the basic requirements of the linear array CCD, students are interested in the use of linear array CCD for bar code recognition and measurement applications. Therefore, in the case of there is not fully equipped with the corresponding special accessories, students find bar code, learn bar code coding and scanning and other principles and design experimental optical path by themselves. Through design and operate the whole process by themselves, not only firmly grasp the CCD image sensor basic principles and knowledge, extend the basic knowledge associated with the bar code. And more importantly, The students have a sense of understanding and clear analysis of ideas in bar code acquisition and identification of the various factors in the process, master the use of linear array CCD to complete the application of bar code scanning, expand the professional knowledge in commodity circulation and other daily life in the actual application.

\subsection{Try free and open experiment to fully mobilize students' enthusiasm for learning}

To maximize the use and development of photovoltaic experimental platform resources to encourage students according to their own interests and hobbies. Based on the existing experimental platform conditions and the completion of compulsory experiments, to design their own experimental content and explore the experimental method of interest and possible applications. This way can give full play to students' innovative thinking, so that students learn from passive to active. For example, the existing optoelectronic integrated experimental platform is equipped with a grating for demonstrating Moiré fringes, consisting of two overlapping metering gratings. In general, the grating's black and white lines are equally wide, i.e., the pitch (grating constant) of the grating is equally spaced. Moiré fringes are generated when the two gratings are close to each other. From the shape of the stripes can be seen, the Moiré fringe position in the two grating engraved angle $\theta$ angle bisector. When the two gratings move relative, the Moiré fringes move in the vertical direction of the raster movement, i.e., the bisector of the angle $\theta$. Moiré fringes move an interval, after one raster move one raster. Therefore, as long as the number of stripes to be measured, you can calculate the amount of displacement of the grating. This is the principle of grating Moiré fringes for dimensional precision measurements. Moiré fringes make the geometric size measurement accuracy almost close to sub-nanometer. To achieve the completion of the grating Moiré fringe high-precision photoelectric detection experiments with the need for surface array $\mathrm{CCD}$ as a detector. But the existing experimental platform is only equipped with a linear array CCD detector, surface array CCD. In order to improve students' awareness of the characteristics and application of grating and grating Moiré fringe, in addition to obtaining sensibility through the demonstration experiment produced by observing Moiré fringes, students are encouraged to use the existing conditions to further explore the relevant knowledge. Students design to use the linear array

$\mathrm{CCD}$ detector and grating with the composition of the experimental system, you can measure the 
grating pitch and the width of the Moiré fringes. Through this experiment, although the students did not measure the exact geometric size of the micro-displacement, but through this experiment students can master the basic principles of grating, understand grating is a important role in the geometric precision measurement technology. Therefore, the open experimental platform and environment, not only make full use of and development of the photoelectric experimental platform resources, more meaningful is to play the students innovative thinking and creativity, greatly play the subjective initiative of students to learn. It can receive unexpected experimental results.

\subsection{Appropriate use of exploratory teaching}

Guide students to think on selected topics, let students acquire knowledge autonomously and use knowledge to solve problems. For example, in teaching the chromaticity diagram allows students to group as a unit, observe and discuss the intuitive understanding on the visual diagram of the intuitive understanding respectively. And then put forward any one point in the figure, let the group discuss the wavelength of this point, the proportion of the three primary colors and their purity. Finally comment on the views of the students and give the correct explanation. It is true that the optoelectronic synthesis experiment is only a modern institution of higher education to carry out. And engineering training is to include turning, milling, grinding, gear processing, boring $\mathrm{CNC}$ car, $\mathrm{CNC}$ milling, machining center, EDM wire cutting, electrician, electronics, fitter, welding, casting, stamping and many other types of work. In the experimental teaching of higher education, not all teaching content is suitable for project teaching and inquiry teaching. Therefore, in teaching it should try to use a variety of and appropriate teaching methods to achieve the best results. At the same time to promote the combination of multiple types of work, so that students participate in challenging innovative projects, such as subject competition, teacher research topics, so that students play a personality and innovative spirit, develop students comprehensive engineering capacity and innovation further.

\subsection{To use experimental projects as a means to consolidate and deepen understanding}

In the class, it should use some experimental phenomena, to find some topic that students familiar with and the topic related to the class, making the classroom atmosphere active to increase student interest and attract their attention. For example, in learning and testing luminous flux and luminous intensity, we can carry a LED lights and energy-saving lamps, power on light, so that students can observe the range of its luminous. And guide students to think and compare the luminous flux and luminous intensity of the concept. And then it can be combined with hands-on test. It can let students to learn the test method, but also to deepen their understanding of the concept. In teaching, it can not be the same as the conventional teaching, regardless of any external factors during the class, in full accordance with the progress of the pre-prepared course. But we should first observe the object of the class, according to the students of the original knowledge system for preparation. During the class, according to different circumstances appropriate adjustments to the content and progress of the class, pay attention to cultivating students' thinking ability and self-learning ability. Guide students to constantly think and innovate. For example, when teaching basic knowledge, the student is ambiguous about the relationship between light intensity, brightness, and illumination at first. Therefore, we choose to start from the three measuring instruments, divide into a small task to guide students to determine the relationship between the three measuring instruments through the completion of experimental measurement tasks. 


\section{Conclusion}

Through the establishment of a more flexible and scientific open experimental conditions and the environment, to stimulate students and teachers innovative ideas, mobilize the enthusiasm of students to take the initiative to develop and improve the ability to think diligently and improve students' creative ability and overall quality. After practicing on the open experimental platform, students can not only master the basic working principle, characteristics and basic parameters of the optoelectronic devices, as well as the basic experiment purpose and requirements of the correct use and measurement methods, carry out the comprehensive experiment training step by step. And even get the creative ability of independent experimental exercise. This is conducive to the combination of optical experiment and photoelectric test comprehensive application ability, optoelectronics, optical communications and information technology. It has a practical application of complex high-level specialized personnel training.

\section{REFERENCES}

[1] MAO.H.J, LIU.J, LIANG.Y.H, HUANG.Z.F, The Operation Mode and Administration in the Comprehensive Laboratory of Optoelectronic Technology for Graduate Students, R ESEAR CH AND EXPLORATION IN LABORATORY.35(9),162-164(2016).

[2] SONG.L.P, Teaching Design of Photoelectric Effect Experiment Based on Quality Cultivation, Experiment Science and Technology.14(3).22, 30-32(2016).

[3] YANG.R, Exploration of cultivating creative ability by photoelectric experiment, Journal of Yunnan University.36(s2), 213-216(2014).

[4] Han.Y.L, Guo.X.J, Ren.J.C, Constructing comprehensive experimental platform with photoelectric system to adapt development of military equipment, Experimental Technology and Management.30(5),102-107(2013).

[5] Chen.H.M, Fan.H, QIAN.C, Shi.W.H, Xu.N , Sun.X.Y, Research and practice of optoeletronic information laboratory construct, OPTICAL TECHNIQUE. 33, 307-308 (2007). 